\title{
符号回归机器学习发现简易催化描述子
}

刘忠范

北京大学化学与分子工程学院, 北京 100871

\section{Simple Catalysis Descriptor Discovered by Symbolic Regression}

\section{Zhongfan Liu}

College of Chemistry and Molecular Engineering, Peking University, Beijing 100871, China.

Email: zfliu@pku.edu.cn

Published online: July 24, 2020.

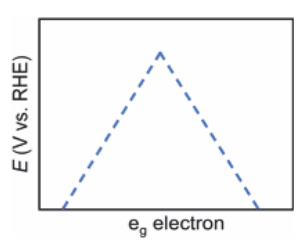

Human Knowledge

DFT calculation required

Volcano scaling

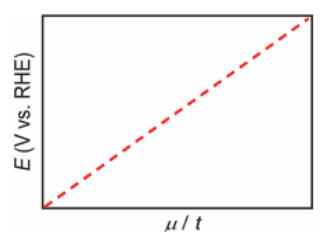

Machine Learning

easily accessible

Linear scaling

experimental verification

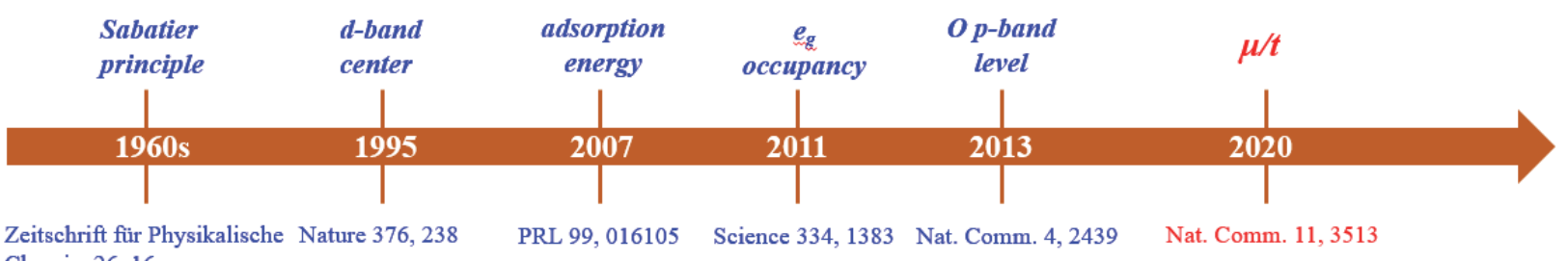

Chemie. 26, 16

氧化物钻钛矿析氧反应 (OER)描述子的发展---从领域知识到机器学习。

描述子(descriptor) 是描述复杂现象的简单标 度。在催化领域, 科学家们数十年来一直在寻找简 单而准确的描述子, 试图定量描述复杂的催化现 象。这些描述子包括但不限于 $d$ 能带中心, 吸附自 由能, 形成焓等。一般来说, 催化活性和这些描述 子定量上呈现火山图(volcano curve)的关系。这是 因为这些描述子的提出均是基于 Sabatier Principle一反应物(中间态)与催化剂之间的吸附能 不能太强, 也不能太弱 1 。

Sabatier Principle与火山图的概念同样被应用 到氧化物钙钛矿析氧反应(OER)的研究中。2011 年, 麻省理工学院Yang Shao-Horn研究组提出了过 渡金属原子 $e_{\mathrm{g}}$ 轨道占据数作为OER描述子, 并据此 发现 $\mathrm{Ba}_{0.5} \mathrm{Sr}_{0.5} \mathrm{Co}_{0.8} \mathrm{Fe}_{0.2} \mathrm{O}_{3}$ (BSCF) 催化剂 ${ }^{2}$ 。此后, 诸 如自由能 ${ }^{3}, \mathrm{O} p$ 带位置 $^{4}$, 过渡金属与 $\mathrm{O}$ 的共价相互
作用 5 等描述子被相继提出。这些描述子基于人类 物理和化学知识的经验总结, 取得了一定的成功。 然而, 它们都需要密度泛函理论(DFT)计算。而 DFT计算不仅耗时耗力, 对诸如 $d$ 带中心, $e_{\mathrm{g}}$ 轨道 占据数等计算还依赖于计算设置(表面自旋态、 $3 d$ 轨道on-site Coulomb U值选取等) ${ }^{6}$, 从而不可避免 地在结果中引入人为因素。因此, 传统的描述子并 不利于大规模材料设计与節选。

近日, 苏州大学能源学院、能源与材料创新研 究院尹万健研究组以氧化物钻铁矿 OER为例, 提 出利用符号回归机器学习方法, 跳过DFT计算, 直 接建立催化活性与材料参数的构效关系 7 。这里的 材料参数指的是组成元素化学配比、离子半径、电 负性、价态、过渡金属离子 $d$ 电子数等在教科书中 查表可得的基本参数。他们的实验合作者美国托 莱多大学Yanfa Yan教授研究组合成了18种已知 
的氧化物钙钛矿, 以产生具有一致性和可比较的 数据集。基于该数据集, 尹万健研究组采用符号回 归方法寻找构效关系表达式, 然后在这些表达式 中选择一个简单而准确的描述子 $\mu / t$, 其中 $\mu\left(r_{\mathrm{B}} / r_{\mathrm{O}}\right)$ 和 $t\left(\frac{r_{\mathrm{A}}+r_{\mathrm{O}}}{\sqrt{2}\left(r_{\mathrm{B}}+r_{\mathrm{O}}\right)}\right)$ 分别是钙钛矿 $\mathrm{ABO}_{3}$ 的八面体因 子和容忍因子。它们只是离子半径的函数, 不需 要DFT计算, 因而更利于大规模材料设计与篮选。 新描述子 $\mu / t$ 表明 $\mathrm{ABO}_{3}$ 中 $\mathrm{A}$ 离子越大, $\mathrm{B}$ 离子 越小, 催化活性越高。据此, 他们设计用大的碱金 属离子占据A位, 小的过渡金属离子占据B位, 以 摩尔组分 0.25 为间隔构造合金, 共产生三千多种可 能的新材料。并进一步通过新描述子 $\mu / t$, 同时兼顾 元素和组分的多样性, 从中选择了13种理论催化 性能最高的材料进行合成。Yan实验组成功合成了 5 种, 发现其中 4 种新材料 $\left(\mathrm{Cs}_{0.4} \mathrm{La}_{0.6} \mathrm{Mn}_{0.25} \mathrm{Co}_{0.75} \mathrm{O}_{3}\right.$ 、 $\mathrm{Cs}_{0.3} \mathrm{La}_{0.7} \mathrm{NiO}_{3} 、 \mathrm{SrNi}_{0.75} \mathrm{Co}_{0.25} \mathrm{O}_{3}$ 和 $\mathrm{Sr}_{0.25} \mathrm{Ba}_{0.75} \mathrm{NiO}_{3}$ ) 的催化活性均高于之前的 18 种, 同时也满足描述 子 $\mu / t$ 的规律。同时, 该描述子还可以被Shao-Horn 研究组2011年Science论文的独立实验数据所证 实 ${ }^{2}$ 。尹等的工作评审过程中, 审稿人(Reviewer \#3) 发现自己研究组积累的数据同样满足新描述子 $\mu / t$ 的线性规律(审稿意见见原文 7 链接)。这些事实表 明描述子 $\mu / t$ 对氧化物钙铁矿 OER 具有普适性。

该工作中简易描述子 $\mu / t$ 的发现得益于符号回 归机器学习方法的运用。一方面, 基于传统的物理 与化学知识, 很难建立催化活性与离子半径的关 系; 另一方面, 虽然机器学习已被广泛运用于材 料、化学等领域, 但其 “黑箱(black-box)” 模型常 被认为不能发现新的 “物理规律”, 而只是对数据 采取一种未知方式的统计拟合, 限制了其广泛应 用 8 。特别是对大多数不熟悉机器学习算法的实验 工作者, 机器学习指导实验存在事实上的困难。符 号回归是一种可解释的机器学习方法 9,10 , 能够提 供目标函数和自变量特征参数之间具体的数学表 达式, 变机器学习 “黑箱” 模型为 “白箱” (glassbox), 为材料设计提供直接指导。尽管潜力巨大,
但在材料与化学科学领域的应用目前却很少。尹 万健等的工作提供了符号回归在材料领域的一个 应用实例, 将激发更多的材料与化学研究者关注 和利用符号回归机器学习加速新材料的发现。

上述研究工作近期在Nature Communications 上在线发表 ${ }^{7}$ 。

\section{References}

(1) Sabatier, P. Berichte der Dtsch. Chem. Gesellschaft 1911, 44 (3), 1984. doi: 10.1002/cber.19110440303.

(2) Suntivich, J.; May, K. J.; Gasteiger, H. A.; Goodenough, J. B.; ShaoHorn, Y. Science 2011, 334 (6061), 1383. doi: $10.1126 /$ science. 1212858 .

(3) Man, I. C.; Su, H.-Y.; Calle-Vallejo, F.; Hansen, H. A.; Martínez, J. I.; Inoglu, N. G.; Kitchin, J.; Jaramillo, T. F.; Nørskov, J. K.; Rossmeisl, J. ChemCatChem 2011, 3 (7), 1159. doi: $10.1002 /$ cctc. 201000397.

(4) Grimaud, A.; May, K. J.; Carlton, C. E.; Lee, Y. -L.; Risch, M.; Hong, W. T.; Zhou, J.; Shao-Horn, Y. Nat. Commun. 2013, 4 (1), 2439. doi: $10.1038 /$ ncomms3439.

(5) Simböck, J.; Ghiasi, M.; Schönebaum, S.; Simon, U.; de Groot, F. M. F.; Palkovits, R. Nat. Commun. 2020, 11 (1), 652. doi: 10.1038/s41467-020-14305-0.

(6) Jacobs, R.; Hwang, J.; Shao-Horn, Y.; Morgan, D. Chem. Mater. 2019, 31 (3), 785. doi: 10.1021/acs.chemmater.8b03840.

(7) Weng, B.; Song, Z.; Zhu, R.; Yan, Q.; Sun, Q.; Grice, C. G.; Yan, Y.; Yin, W. -J. Nat. Commun. 2020, 11 (1), 3513. doi: 10.1038/s41467-020-17263-9.

(8) Vellido, A.; Martín-Guerrero, J.; Lisboa, P. J. Making Machine Learning Models Interpretable. In Proc ESANN; 2012; Vol. 12, pp. 163-172. doi: 10.1.1.431.5382.

(9) Ouyang, R.; Curtarolo, S.; Ahmetcik, E.; Scheffler, M.; Ghiringhelli, L. M. Phys. Rev. Mater. 2018, 2 (8), 083802. doi: 10.1103/PhysRevMaterials.2.083802.

(10) Wang, Y.; Wagner, N.; Rondinelli, J. M. MRS Commun. 2019, 9 (3), 793. doi: $10.1557 / \mathrm{mrc} .2019 .85$. 